\title{
The generation of jets from young stars: an observational perspective
}

\author{
T. P. Ray \\ School of Cosmic Physics, Dublin Institute for Advanced Studies, 31 Fitzwilliam Place, \\ Dublin 2, Ireland \\ email: tr@cp.dias.ie
}

\begin{abstract}
The birth of a young star is accompanied not only by accretion but by the expulsion of matter as well in the form of a collimated outflow. These outflows are seen at various wavelengths from X-rays to the radio band, but ultimately the driving mechanism appears to be a highly collimated supersonic jet that contains not only atomic but molecular components as well. These jets may also play a key role in the star formation process itself since they could be one of the primary mechanism for removing angular momentum from the accretion disk thereby allowing accretion to occur. Whereas much is known about their propagation on large-scales (i.e., hundreds of AU to several parsecs) from both observations and simulations, we must explore the "central engine" in order to understand how they are generated. While this is particularly challenging, high spatial resolution studies are beginning to reveal interesting data from which we can confront the various models. In this review, I will summarise what these studies suggest and note how they already favour certain models over others. I will also describe some of the results from spectro-astrometry and interferometry that are revealing details of outflows on milliarcsecond scales from the source.
\end{abstract}

Keywords. Stars: pre-main-sequence, stars: low-mass, brown dwarfs, stars: winds, outflows, ISM: jets and outflows, ISM: Herbig-Haro objects.

\section{Introduction}

The phenomenon of jets from young stars is not only striking but may be fundamental to the star formation process itself. In particular they could be channels for removing angular momentum thereby allowing accretion to occur and thus regulating the final mass of the newborn star. Moreover they may add turbulence, energy and affect the chemical composition of the surrounding molecular cloud (Arce \& Sargent 2004; Li \& Nakamura 2006; Banerjee, Klessen \& Fendt 2007) and therefore play a role in determining conditions in the circumstellar environment. Ultimately, in ways not currently understood, they could be a factor that helps shape the initial mass function (IMF).

Outflows from young stars were first discovered in molecular transitions in the early 1980s (e.g. Snell, Loren \& Plambeck 1980) with the opening of the mm-band window for astronomy. Later it was realised that the optically visible nebulous patches known as Herbig-Haro (HH) objects (Herbig 1950; Haro 1952), uncovered many years before, were part of the same phenomenon. Deep optical images, made possible with the advent of CCD detectors, revealed that many well-known $\mathrm{HH}$ objects were just the brightest parts of a highly collimated jet, visible in low excitation emission lines (Mundt \& Fried 1983; Ray 1987; Bührke, Mundt \& Ray 1988), and that it was the jet that drove the slower gas seen, for example, in low order CO rotational transitions.

In this review, we will concentrate on what the most recent observations have to tell us about the driving mechanism for jets from young stars as well as sub-stellar objects, i.e., brown dwarfs. We will begin by summarising what is known about the 
outflow phenomenon with the emphasis on the highly collimated component be it atomic (ionised or neutral) or molecular $(\S 2)$. The challenges in resolving the central engine from an observational perspective will be explored in $\S 3$ as well as the results of various high spatial resolution studies. Finally in $\S 4$, we will briefly discuss what the techniques of spectro-astrometry and interferometry are beginning to reveal about outflows within a few milliarcseconds from their cores.

\section{The jet phenomenon in context}

The combination of multi-epoch imaging (typically a few years apart) and spectroscopy quickly revealed many of the basic parameters of jets from young stellar objects (YSOs). As with $\mathrm{HH}$ objects, jets reveal themselves through their line emission which derives from radiative cooling in post-shock zones. This line emission is dominated by forbidden transitions, e.g. [OI] $\lambda \lambda 6300,6363$, [SII] $\lambda \lambda 6716,6731$, and [FeII]1.64 $\mu \mathrm{m}$ (e.g. Nisini et al. 2005) although permitted, e.g. H $\alpha$, and semi-forbidden transitions, e.g. CIII]1909 (Böhm \& Böhm-Vitense 1984) are also seen. Intermediate resolution spectroscopy can not only reveal the kinematic state of the jet, through radial velocity studies, but combination of lines can tell us other basic physical parameters such as electron density, $\mathrm{n}_{\mathrm{e}}$ using the [SII] doublet. In addition, if we assume charge exchange, i.e., the ionised state of Oxygen and Nitrogen is determined via charge exchange with Hydrogen, and that Sulphur is singly ionised because of its low IP, other parameters such as electron temperature, $\mathrm{T}_{\mathrm{e}}$, and ionised fraction $\chi$ can be determined (Bacciotti \& Eislöffel 1999). In turn this leads to total density estimates. Since we know the various line fluxes, and as the radiation is optically thin, such fundamental parameters as jet mass loss rates as well as mechanical luminosities can be derived.

Typical (hydrodynamic) Mach numbers $M$ were found to be 10-30, with mass loss rates $\dot{\mathrm{M}}_{\text {jet }} \approx 10^{-8}-10^{-6} \mathrm{M}_{\odot} \mathrm{yr}^{-1}$ and velocities of around $100-300 \mathrm{~km} \mathrm{~s}^{-1}$. In addition although initially it was thought that these collimated outflows were only a few thousand AU in length, it was soon established that many were comparable in size to the parent molecular cloud itself, i.e., parsec-scale (Ray 1987). Such lengths however are to be expected if, as seems to be the case, the outflow phenomenon lasts the same time as the accretion phase (i.e., $\approx 10^{6}$ years for solar mass YSOs) and giving their typical velocities. Moreover, in addition to their remarkable lengths, their degree of collimation, as indicated by the opening angle $\theta_{j e t}$, is striking. Typically this is only a few degrees and close to what is expected if such jets are freely expanding, i.e., $\theta_{\text {jet }} \approx 1 / M$.

Close examination of YSO jets, such as HH 30 (Ray et al. 1996) invariably show they are not continuous but instead consist of a string of knots. On timescales of a few months to years new knots may appear close to the source and these either fade away or blend into existing knots. Shock velocities at the knots are usually low (a few tens of $\mathrm{km} \mathrm{s}^{-1}$ ) and considerably less than the jet velocity itself. This has led to the idea that the knots are due to temporal variations in the flow and are regions were faster jet material rams into slower moving gas ahead of it (e.g. Raga et al. 2007). Since the jet is moving supersonically this gives rise to a two shock structure: at the upstream shock the faster material is decelerated while at the downstream shock the slower material is accelerated. The region in between, known as the working surface, is over-pressured with respect to its environment and some gas is ejected sideways (although note that the gas is radiatively cooling). According to this model we would expect the knots to move outwards with velocities comparable to that of the jet. Such a result is found from proper motion studies (McGroarty, Ray \& Froebrich 2007).

According to the picture painted above, we only see those regions of the jet that are currently being shocked. There must be other parts of the jet that are undisturbed and 


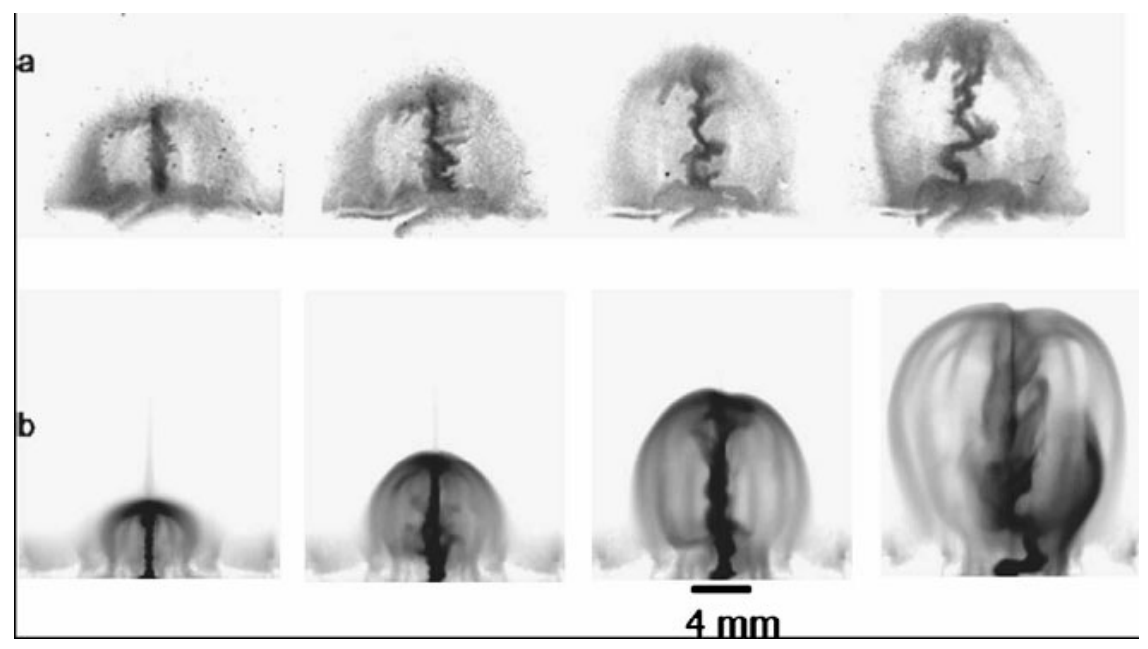

Figure 1. (a) Laboratory jets produced at Imperial College London's z-pinch facility. These jets are magnetically collimated by magnetic hoop stresses. Note the variability in emission, which in this case is probably due to the growth of instabilities, and the physically small scale of these jets. (b) Simulations of the magnetic tower jets seen in (a). Images courtesy of Andrea Ciardi and Sergey Lebedev.

hence invisible. Such regions are revealed however, through photo-ionisation, when the jet is sufficiently close to a UV source, e.g. an O-type star. These so-called irradiated jets can thus be found near HII regions, such as Orion and Carina, and give us more of an idea of what the true outflow history of a young star is like (Bally 2007).

Computer simulations of jets from young stars, at least on extended (0.01-1.0 pc) scales, have now reached a high degree of complexity. Not only do the codes allow for atomic and molecular cooling but also incorporate magnetic fields - the parameter that we perhaps know least in YSO jets (de Colle \& Raga 2006). The problem is ideally done in a parallel processing environment and current codes can also incorporate adaptive mesh refinement (AMR) to track the physically most interesting parts of the flow (Cunningham et al. 2006). Such simulations are useful not only in revealing the expected morphology and properties of the working-surfaces but also in testing how the jet interacts with its environment. For example it has long been recognised that, in regions that are sufficiently dense, the atomic jet "pushes" ambient material to give rise to the slower (V a few tens of $\mathrm{km} \mathrm{s}^{-1}$ ) molecular outflow traced in CO rotational transitions. Simulations can address whether the molecular gas has the expected distribution of mass versus velocity $m(v)$ if it is jet-driven (Downes \& Cabrit 2003).

In recent years however YSO jet simulations have come from an unexpected quarter, laboratories involved in fusion research. It is now possible, using either high power lasers or imploding z-pinch wire arrays, to produce high Mach number jets $(M \sim 5-20)$ at a number of facilities (Lebedev et al. 2004; Stehlé et al. 2005; Sublett et al. 2007). Radiative cooling of the jet, at least for those produced through z-pinching is through X-ray emission, rather than UV, optical and near-infrared lines, but many fundamental numbers (e.g. the ratio of cooling length to dynamical length and the plasma $\beta$ parameter) can be made to match. It is even possible, to introduce jet rotation (Ampleford et al. 2007)! While many of these simulations are at an early stage, the laboratory jets bear a remarkable resemblance to those seen from YSOs (see Figure 1). Perhaps just as importantly they can also be used to benchmark various jet codes, rigorously testing their limitations (Ciardi et al. 2007). 


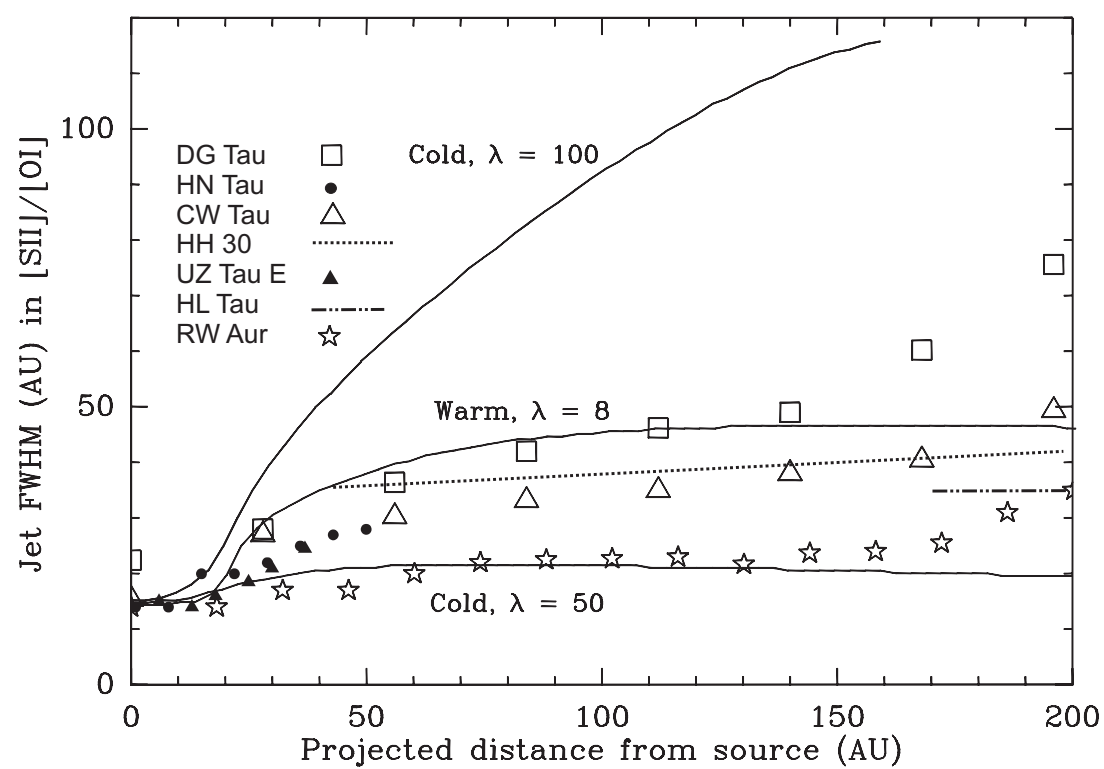

Figure 2. Jet width (FWHM) as a function of distance from the source using [SII] and [OI] images. Solid lines are predicted variations based on cold, low efficiency (high $\lambda$ ) models and warm, high efficiency (low $\lambda$ ) models. Efficiency is measured as the ratio of the mass outflow to the mass inflow (accretion) rates. Although cold solutions can be found to fit the data, the resultant poloidal velocity is much higher than observed favouring warm, moderately efficient, solutions. From Dougados et al. (2004) with additional data from Hartigan et al. (2004).

\section{The challenge of observing the jet engine}

While there is some uncertainty as to the origins of jets (see for example Ferreira, Dougados \& Cabrit 2006 and Shu, this volume) the general consensus is that the jet is initially generated in a region less than $1 \mathrm{AU}$ in diameter and almost certainly arises from the circumstellar disk. Even for the nearest star formation regions, for example Taurus Auriga or Ophiuchus, this scale corresponds to less than 10 milliarcseconds on the sky. Such a small region cannot be observed using conventional ground based instruments although interferometry (see below) is beginning to observe such zones. The situation however is not quite as bad as it initially looks. Almost all models (see, for example, Pudritz et al. 2007) predict jets are accelerated and focussed over larger scales, we thus stand a chance of probing them in the region where they are reaching their asymptotic values (i.e., within $1^{\prime \prime}$ for the nearest star formation regions).

Studying the jet line emission close to the source with sufficient angular resolution usually means observing in the optical. Many jet sources however (e.g. HH1/2 VLA-1) are highly embedded and thus we must turn to a number of Classical T Tauri stars (CTTS) with jets if we are to probe their base. Examples include DG Tau (Dougados et al. 2000), CW Tau and HN Tau (Hartigan, Edwards \& Pearson 2004). Observations of these jets have not only been done from the ground using adaptive optics (AO) but also from space using the novel technique of slit-less spectroscopy (Hartigan et al. 2004). Such observations reveal the variation in jet width as a function of distance from the source (see Figure 2). Comparison with MHD models (Dougados et al. 2004) show that the jets must be launched warm. Although 'cold' solutions can be made to fit (again see Figure 2) they predict too high a poloidal jet velocity in comparison to what is observed.

There are a number of other questions that observations close to the source are beginning to address. For example do molecules and dust survive the jet launching process? 


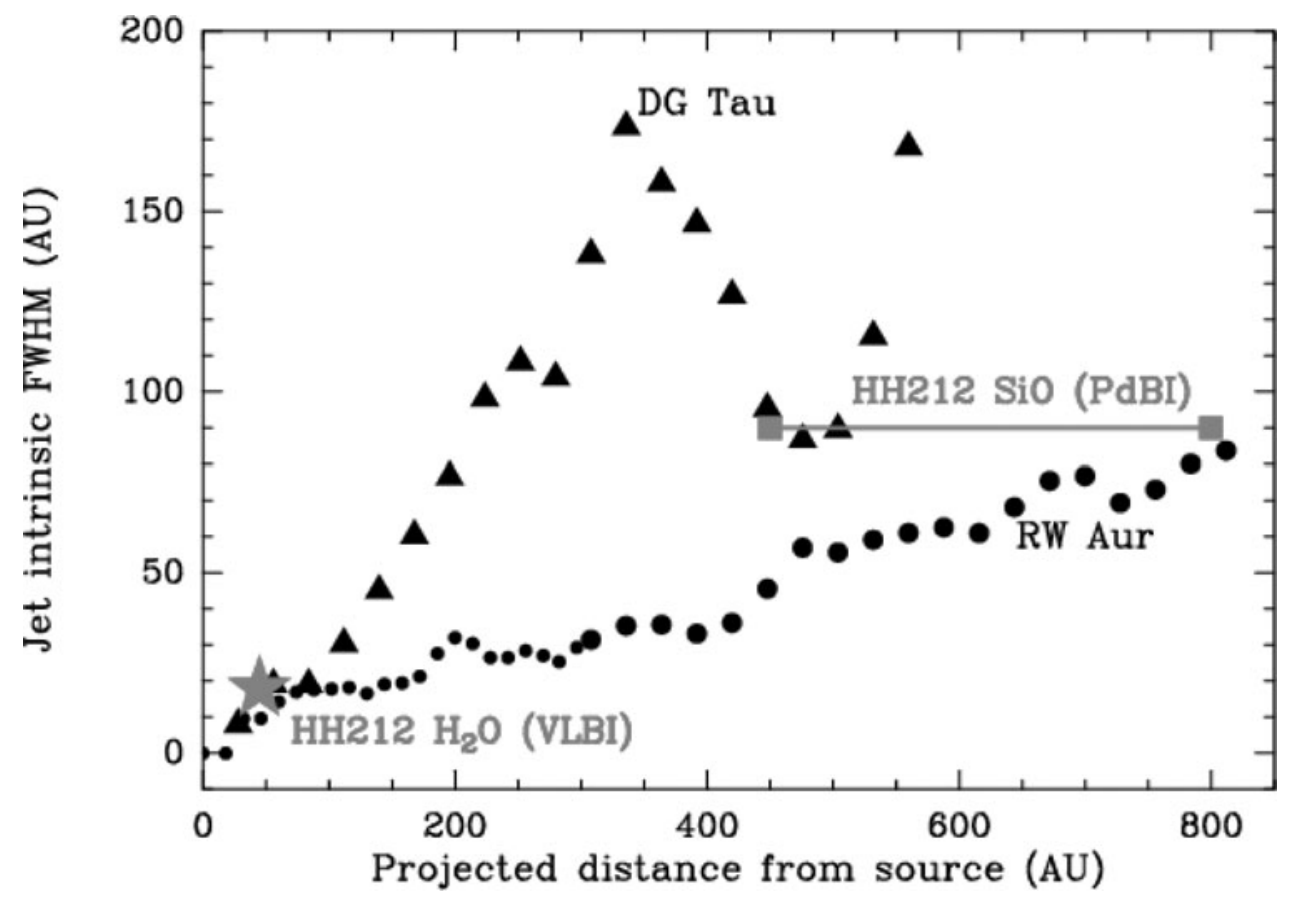

Figure 3. Comparison plot of jet width (FWHM) as a function of distance from the source for DG Tau, RW Aur and HH 212. Whereas the observations for the two classical T Tauri jets were made in optical forbidden lines, those for $\mathrm{HH} 212$ were made in $\mathrm{SiO}$ lines using the Plateau de Bure Interferometer. Widths, at a given distance from the source, are seen to be similar in all cases suggesting $\mathrm{SiO}$ molecules are launched with the jet rather than entrained. The width of the cone defining the $\mathrm{H}_{2} \mathrm{O}$ maser emission of $\mathrm{HH} 212$ is also shown. Note how the masers seem confined to the $\mathrm{SiO}$ jet again suggesting they are part of the initial flow. Figure from Cabrit et al. (2007).

Clearly molecules are part of the outflow as witnessed by the well-known, often bipolar, lobes seen in low order CO rotational transitions. As stated above however this gas is clearly pushed ambient material and has not travelled in towards the young star and been ejected from the disk. The situation is less clear for example with high velocity $\mathrm{CO}$ (which appears much more collimated) seen in higher order transitions, shocked $\mathrm{H}_{2}$ and $\mathrm{SiO}$ emission. Are such molecules entrained in the jet or are they launched with it and therefore constituent part of the initial flow? Recent high spatial resolution SiO observations ( 0 '.34) by Codella et al. (2007) using the Plateau de Bure (PdB) interferometer in its extended configurations certainly suggests that some molecules are launched as part of the jet. HH 212 is a well known outflow with a dramatic large-scale bipolar jet first seen in shocked $\mathrm{H}_{2}$ emission (Zinnecker, McCaughrean \& Rayner 1998). The PdB observations show that the $\mathrm{SiO}$ emission is confined to a highly-collimated bipolar jet (with a FWHM width of 0 .' 35 close to the source) in the same direction as the $\mathrm{H}_{2}$ jet and that it has very similar kinematics to the latter. Such jet widths are comparable to those seen in outflows from classical $\mathrm{T}$ Tauri stars (see Figure 3 and previous Figure). Moreover in the case of $\mathrm{HH} 212$, the $\mathrm{H}_{2} \mathrm{O}$ masers also seem confined to a narrow cone (see Figure 3).

Evidence is also emerging that dust may at least partially survive the launching process. Figure 4 is from Podio et al. (2006). It shows for example in the $\mathrm{HH} 111$ jet, $\mathrm{Ca}$ is depleted close to the source presumably onto dust grains. At larger distances the expected 


\section{Ca and C DEPLETION}

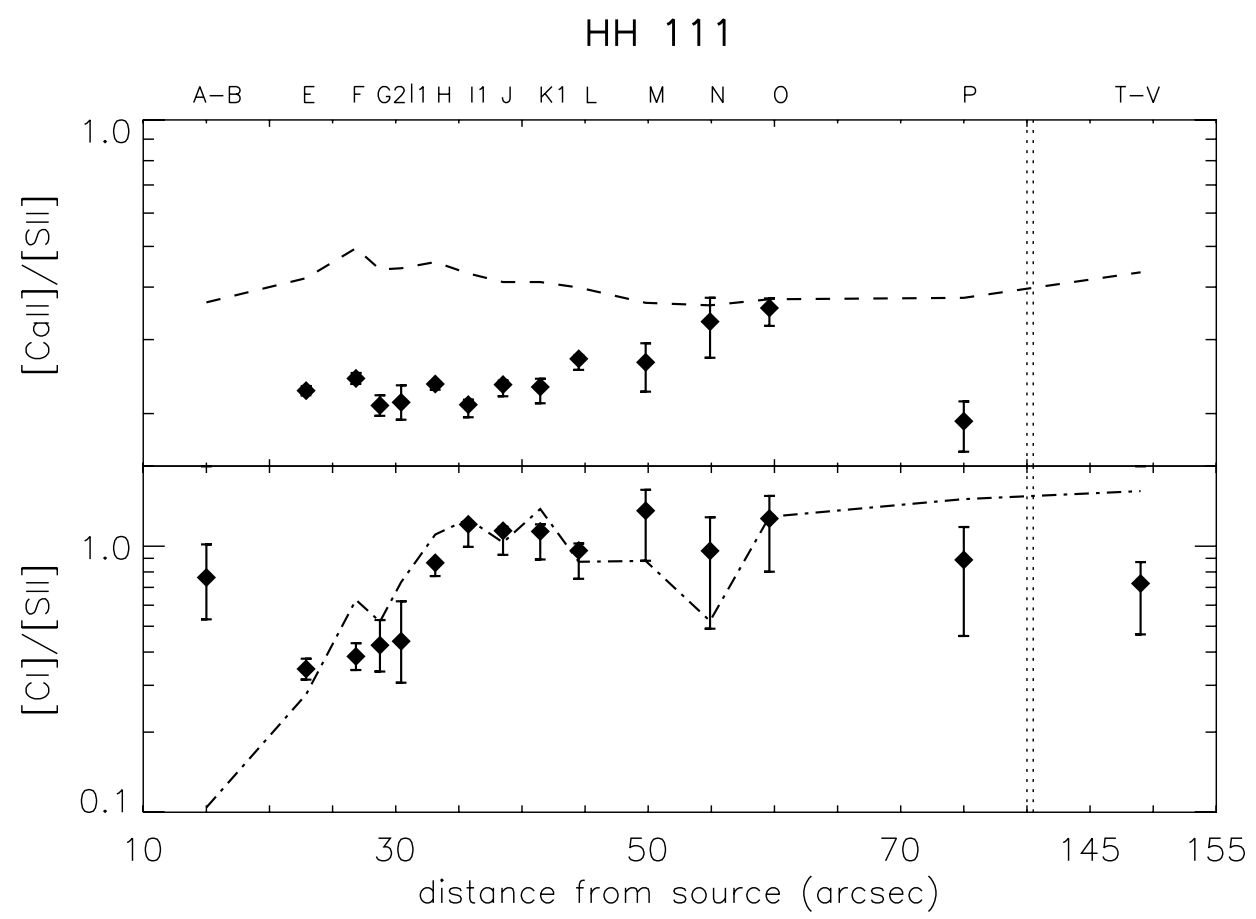

Figure 4. Plots of $[\mathrm{CaII}] /[\mathrm{SII}]$ and $[\mathrm{CI}] /[\mathrm{SII}]$ for the $\mathrm{HH} 111$ jet taken from Podio et al. (2006). These show that $\mathrm{Ca}$ is depleted onto dust grains at least for the initial part of the flow. At larger distances however the dust may be destroyed, presumably as a result of passing through multiple weak shocks in the jet.

abundance of $\mathrm{Ca}$ is recovered perhaps because the dust has passed through a number of shocks before being destroyed. The effect is also seen in HH 34 and in Fe as well as Ca.

One of the most interesting findings in recent years from high spatial resolution studies is that YSO jets may be rotating (Bacciotti et al. 2002; Coffey et al. 2004; Coffey et al. 2007; Ray et al. 2007). Of course MHD models, which assume jet material is launched centrifugally along magnetic field lines, expect jets to rotate (Pudritz et al. 2007). The difficulty in detecting such rotation is that at distances of about $200 \mathrm{AU}$ from the source, corresponding to $1-2^{\prime \prime}$ for the nearest star formation regions, we expect rotational velocities of around 10-20 $\mathrm{km} \mathrm{s}^{-1}$. This compares to jet (poloidal) velocities that are typically 10 times larger. Moreover as the jet widens, with increasing distance from the source, rotational velocities are expected to decrease even further in order to conserve angular momentum.

The earliest observations to discover possible rotation close to the source were made using the Space Telescope Imaging Spectrograph (STIS) (Bacciotti et al. 2002). A series of overlapping narrow $(0.11)$ slits were placed parallel to the DG Tau jet. Using data from these observations it was then possible to reconstruct "images" of the jet not only in individual optical lines but also in different velocity channels. Interestingly differences in radial velocity were observed between opposing slit pairs on either side of the outflow axis. These differences were typically found to be 5 to $25 \mathrm{~km} \mathrm{~s}^{-1}$ with errors of around $\pm 5 \mathrm{kms}^{-1}$, i.e., more or less in line with expected values based on models that launch the jet from extended parts of the disk (around 0.1-1 AU). 
DG Tau approaching jet

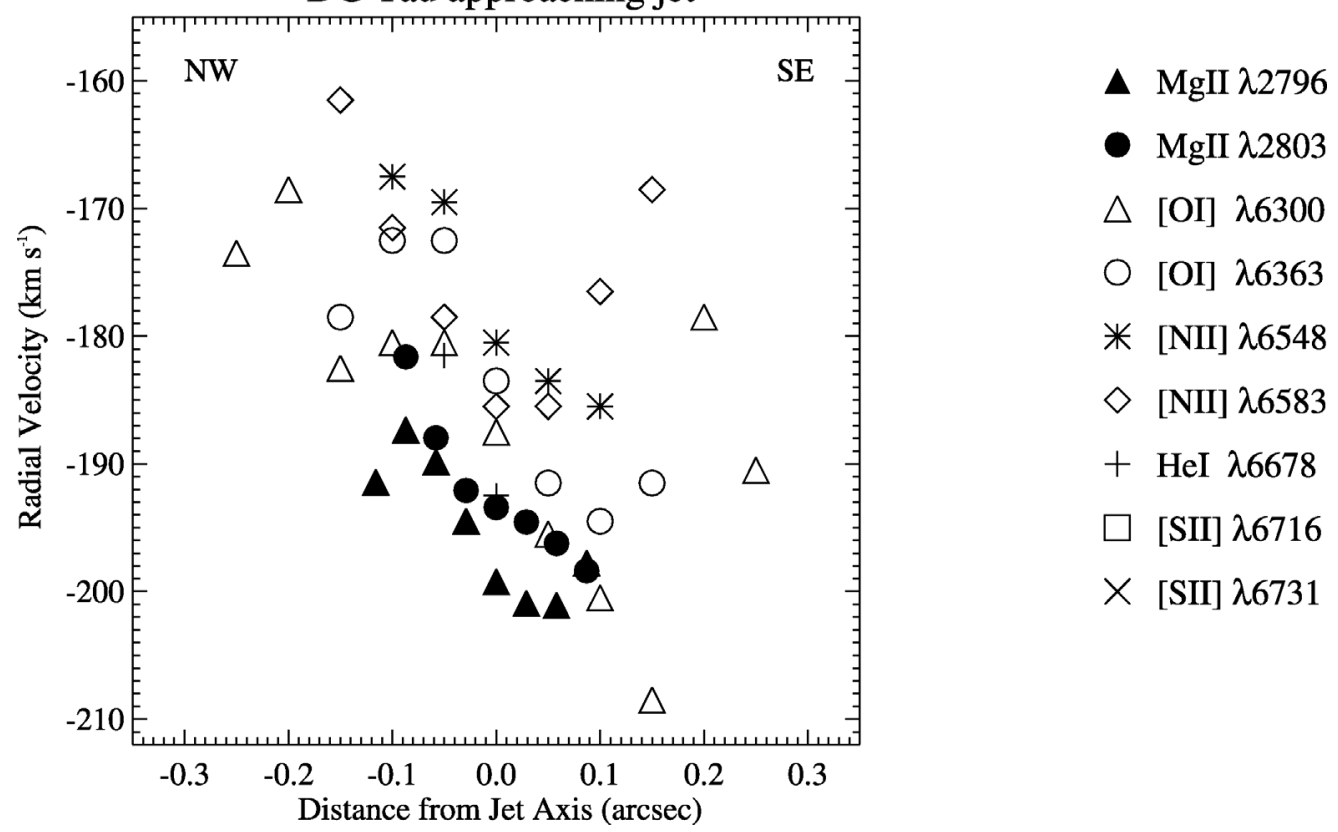

Figure 5. Comparison of observed radial velocities, in optical and UV lines, across the DG Tau blueshifted jet as a function of distance from the jet axis. The Mg UV lines are filled in circles and triangles (see key). Note the consistency between the optical and UV data. Plot taken from Coffey et al. (2007).

Further confirmation of the observed latitudinal velocity differences came from UV line studies again using STIS (Coffey et al. 2007). The magnitude of the differences were seen to be similar to those observed optically (see Figure 5). Velocity differences, again compatible with jet rotation, have also been seen from the ground in the $\mathrm{H}_{2} 2.12 \mu \mathrm{m}$ line (Chrysostomou et al. 2007, in preparation).

In almost all cases, consistency is seen in the system as a whole, e.g. the disk and jet lobes of HH 212 and DG Tau rotate in the same sense. This is as one might expect if the jet is launched from a rotating disk. Moreover in the case of bipolar jets, e.g. RW Aur, both red and blueshifted jets are seen to rotate in the same sense, i.e., with opposite helicity. Again this is as expected. There appears however to be a difficulty in one case: the disk of the RW Aur jet seems to rotate in the opposite sense to its bipolar jet (Cabrit et al. 2006). This system is a hierarchical one and thus it dynamics may be complicated. Nevertheless further study is required to understand this anomaly.

To summarise jets from young stars (both the atomic and molecular components) seem to rotate. Since line diagnostics can also be used to derive mass outflow rates, momentum transfer rates, etc., rotational velocities estimates can be used in conjunction with this data to derive approximate angular momentum transfer rates (e.g. Bacciotti et al. 2004). The values derived are consistent with most of the angular momentum, necessary to maintain accretion in the disk, being transported by the jets. Note however that different models have varying levels of jet angular momentum transport: extended disk-wind models have higher specific angular momentum per unit mass than $\mathrm{X}$-wind models. The derived values tend to favour the former over the latter. 


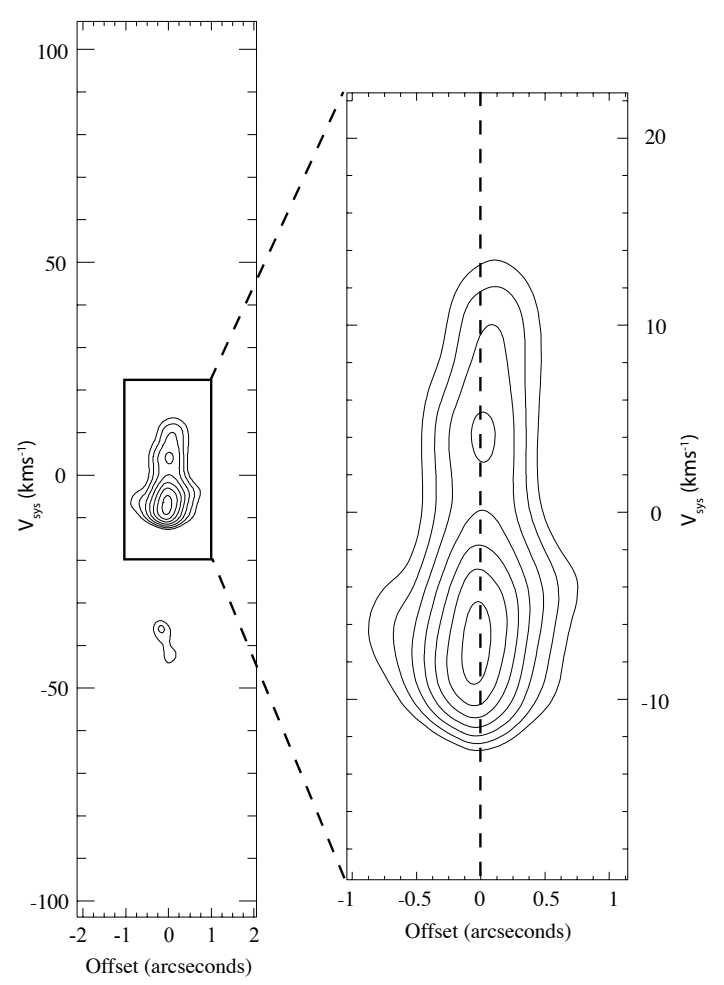

Figure 6. Position Velocity (PV) diagram in the vicinity of the $[\mathrm{OI}] \lambda 6300$ line for the 24 Jupiter mass young brown dwarf 2MASSW J1207334-393254. The data were taken with UVES on the VLT. Velocities are systemic. Both blue and redshifted emission is seen spatially offset either side of the continuum indicating the presence of a bipolar outflow. The offsets are more evident in the spectro-astrometric plot of Figure 7. Plot is from Whelan et al. (2007).

\section{Spectro-astrometry and interferometry}

In recent years the specialised technique of spectro-astrometry has been used to derive data about outflows on scales less than the seeing-disk. The method is explained in detail in Bailey (1998) and Porter, Oudmaijer, \& Baines (2004) as a means of separating close binaries, so I will only briefly describe it here.

Although the image of a star is smeared into a disc comparable to the seeing, the accuracy with which we can determine the centroid of the disc, i.e., the position of the star, is a function of the number of collected photons $N_{p}$. More precisely

$$
\sigma_{\text {Centroid }}=\frac{\operatorname{Seeing}(\text { mas })}{2.3548 \sqrt{N_{p}}}
$$

Thus for $N_{p}=10^{6}$, and seeing around $1^{\prime \prime}, \sigma_{\text {Centroid }} \approx 1$ mas.

Now suppose we have a long-slit spectrum of a YSO which in addition to the continuum from the star, also contains a $\mathrm{HH}$ knot close to its source, i.e., well within the seeing disc. Then a centroid fit to the continuum, as a function of wavelength, will deviate at the lines emitted by the $\mathrm{HH}$ object. In effect the fit is dragged by the latter. Assuming no a priori knowledge of the outflow direction, two preferably orthogonal slit positions are required to specify the direction of the $\mathrm{HH}$ emission with respect to its source (note that the $\mathrm{HH}$ emission is contained within the slit). 

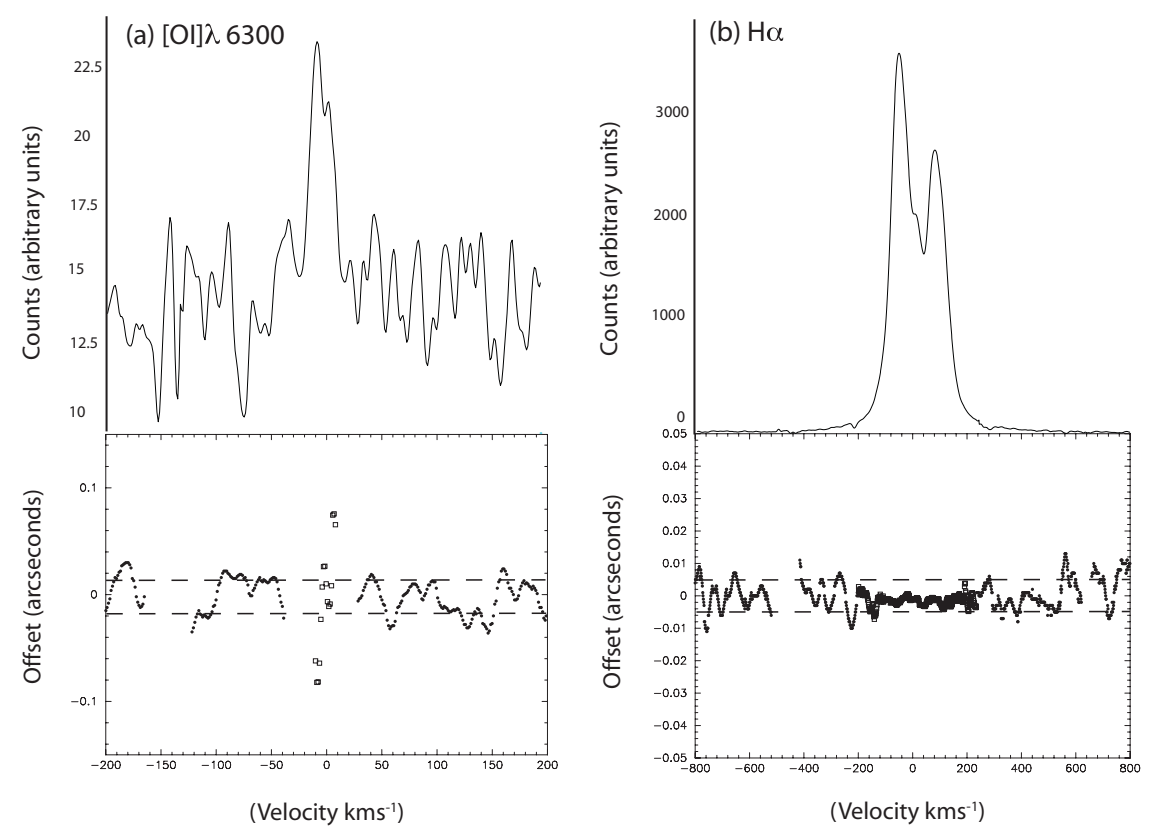

Figure 7. Spectro-astrometric plots for the young brown dwarf 2MASSW J1207334-393254. The $[\mathrm{OI}] \lambda 6300$ line (a) is on the left and $\mathrm{H} \alpha$ (b) on the right. Top panels show line profiles and bottom panels the corresponding offset from the continuum against velocity. Note how the blue and red [OI] wings are offset in the negative and positive direction respectively. In comparison no offset is seen in the $\mathrm{H} \alpha$ line as expected if most of the line originates from accretion.

As the emission we are interested in is within the seeing disc, or more precisely the point spread function (PSF) since the method is obviously applicable to observations from space, the amount of information we can recover is limited. Nevertheless the technique has been used to:

(a) Reveal what parts of a line are due to an "extended outflow" as opposed to magnetospheric activity close to the star (Whelan, Ray \& Davis 2004),

(b) Determine the outflow direction for $\mathrm{HH}$ emission that is very close to its source, using either orthogonal slits or an integral field unit,

(c) Uncover disk dust holes by being able to distinguish the red and blueshifted jets close to the star in permitted lines but not in forbidden lines (e.g. Takami, Bailey \& Chrysostomou 2003). This is because the forbidden emission comes from more extended (distant from the source) regions of the jet where the critical electron density is sufficiently low,

(d) Detect outflows from young brown dwarfs (see below).

An example of the power of the technique is the recent detection of outflows from young brown dwarfs (Whelan et al. 2005; Whelan et al. 2007). Figure 6 shows a long-slit spectrum, in the region of the [OI] 26300 line, taken with UVES on the VLT of the 24 Jupiter mass young brown dwarf 2MASSW J1207334-393254. The total exposure time was 5 hours. The velocity scale is systemic and both blue and redshifted line emission can be seen. The red and blueshifted emission is spatially offset either side of the continuum position (marked as a dashed line in the figure). This is even more evident in the spectro-astrometric plot shown in Figure 7 which shows the offsets $(\approx 0$ '” 1$)$ of the [OI] line 


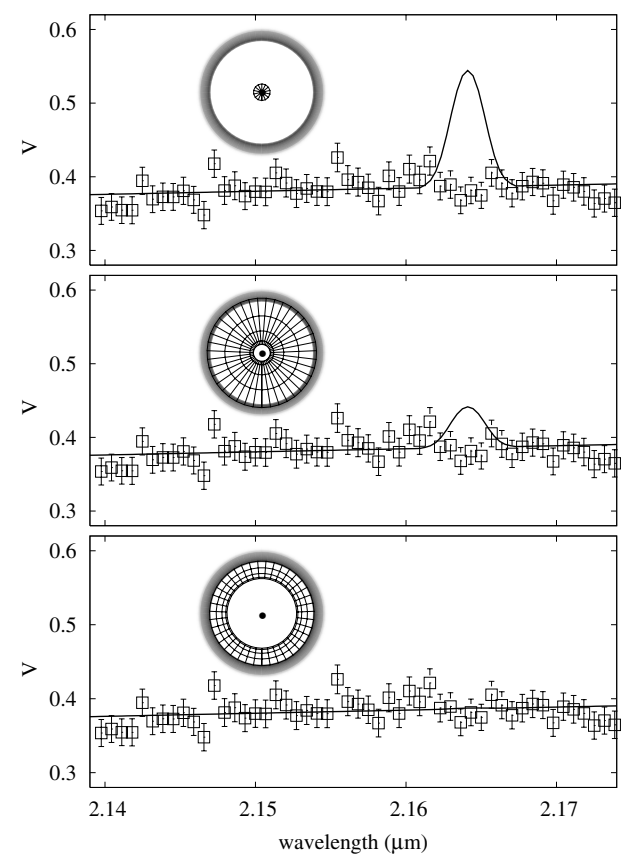

Figure 8. Br $\gamma$ emission visibility curves from HD 104237 based on data from AMBER/VLTI. Data points are empty squares with error bars. Model fits are solid lines. Three different models are shown in the panels. The upper one is for a magnetospheric accretion model in which the $\mathrm{Br} \gamma$ emission arises from inside the co-rotation radius, i.e., very close to the star. The middle panel assumes the emission comes from the co-rotation radius out to $0.45 \mathrm{AU}$. Finally the bottom panel, which is the best fit, assumes the emission comes from 0.2 to $0.5 \mathrm{AU}$. Figure from Tatulli et al. (2007).

displaced on either side of the continuum as we pass through its blue and red wings. In contrast to the forbidden emission, most of the $\mathrm{H} \alpha$ line is thought to arise from accretion very close to the BD (Natta et al. 2004). This is confirmed in the spectro-astrometric plot (Figure 7) which shows no offset in the $\mathrm{H} \alpha$ line. In fact the scatter is even less than in the continuum itself due to the high $\mathrm{S} / \mathrm{N}$ ratio of the line.

2MASSW J1207334-393254 appears to be the lowest mass galactic object known with a jet and suggests that even Jupiter mass planets may drive jets when forming. One other remark worth making is that the radial velocity of this outflow appears to be low. This is consistent with the fact that Scholz, Jayawardhana \& Brandeker (2005) consider 2MASSW J1207334-393254 to have an edge-on disk and thus the outflow should almost be in the plane of the sky.

A number of optical/near-infrared interferometers are either coming on-stream, such as AMBER/VLTI (Petrov et al. 2007), or will be available soon, like the LBT Interferometer (Herbst 2003). Although it is early days, first results are very encouraging and already provide very interesting information. An example is illustrated in Figure 8 which shows the AMBER/VLTI visibility curve against model fits for the origin of the wind from the young Herbig Ae star HD 104237. Full details are given in Tatulli et al. (2007) but essentially the interferometer results show the $\mathrm{Br} \gamma$ emission must originate from a zone 0.2 to $0.5 \mathrm{AU}$ from the star, i.e., it is consistent with the wind being launched from an extended region of the accretion disk. Such distances are an order of magnitude greater than expected from the X-wind model (Shu et al. 2000) according to which most of the 
$\operatorname{Br} \gamma$ emission should arise from close to the co-rotation radius. Finally it should be noted that HD 104237 is a jet source (Grady et al. 2004).

\section{Acknowledgements}

I wish to acknowledge support through the Marie Curie Research Training Network JETSET (Jet Simulations, Experiments and Theory) under contract MRTN-CT-2004005592 and from Science Foundation Ireland under contract 04/BRG/P02741. I also would like to thank Immo Appenzeller and Jerome Bouvier for their patience in awaiting this manuscript.

\section{References}

Ampleford, D. J., et al. 2007, Astrophys. Space. Sci., 307, 51

Arce, H. G., \& Sargent, A. I. 2004, Astrophys. J., 612, 342

Bacciotti, F., Ray, T. P., Mundt, R., Eislöffel, J., \& Solf, J. 2002, Astrophys. J., 576, 222

Bacciotti, F., \& Eislöffel, J. 1999, Astron. \& Astrophys., 342, 717

Bacciotti, F., Ray, T. P., Coffey, D., Eislöffel, J., \& Woitas, J. 2004, Astrophys. Space. Sci., 292, 651

Bailey, J. 1998, Mon. Not. Roy. Astron. Soc., 301, 161

Bally, J. 2007, Astrophys. Space. Sci., 279

Banerjee, R., Klessen, R. S., \& Fendt, C. 2007, Astrophys. J., in press

Böhm, K. H., \& Böhm-Vitense, E. 1984, Astrophys. J., 277, 216

Bührke, T., Mundt, R., \& Ray, T. P. 1988, Astron. ES Astrophys., 200, 99

Cabrit, S., Pety, J., Pesenti, N., \& Dougados, C. 2006, Astron. \&6 Astrophys., 452, 897

Cabrit, S., Codella, C., Gueth, F., Nisini, B., Gusdorf, A., Dougados, C., \& Bacciotti, F. 2007, Astron. \& Astrophys., 468, L29

Ciardi, A., et al. 2007, Astrophys. Space. Sci., 307, 17

Codella, C., Cabrit, S., Gueth, F., Cesaroni, R., Bacciotti, F., Lefloch, B., \& McCaughrean, M. J. 2007, Astron. \& Astrophys., 462, L53

Coffey, D., Bacciotti, F., Woitas, J., Ray, T. P., \& Eislöffel, J. 2004, Astrophys. J., 604, 758

Coffey, D., Bacciotti, F., Ray, T. P., Eislöffel, J., \& Woitas, J. 2007, Astrophys. J., 663, 350

Cunningham, A. J., Frank, A., Quillen, A. C., \& Blackman, E. G. 2006, Astrophys. J., 653, 416

de Colle, F., \& Raga, A. C. 2006, Astron. Es Astrophys., 449, 1061

Dougados, C., Cabrit, S., Lavalley, C., \& Ménard, F. 2000, Astron. \& Astrophys., 357, L61

Dougados, C., Cabrit, S., Ferreira, J., Pesenti, N., Garcia, P., \& O'Brien, D. 2004, Astrophys. Space. Sci., 292, 643

Downes, T. P., \& Cabrit, S. 2003, Astron. ES Astrophys., 403, 135

Ferreira, J., Dougados, C., \& Cabrit, S. 2006, Astron. \& Astrophys., 453, 785

Grady, C. A., et al. 2004, Astrophys. J., 608, 809

Haro, G. 1952, Astrophys. J., 115, 572

Hartigan, P., Edwards, S., \& Pierson, R. 2004, Astrophys. J., 609, 261

Herbig, G. H. 1950, Astrophys. J., 111, 11

Herbst, T. 2003, Astrophys. Space. Sci., 286, 45

Lebedev, S. V., et al. 2004, Astrophys. J., 616, 988

Li, Z.-Y., \& Nakamura, F. 2006, Astrophys. J., 640, L187

McGroarty, F., Ray, T. P., \& Froebrich, D. 2007, Astron. \& Astrophys., 467, 1197

Mundt, R., \& Fried, J. W. 1983, Astrophys. J., 274, L83

Natta, A., Testi, L., Muzerolle, J., Randich, S., Comerón, F., \& Persi, P. 2004, Astron. \& Astrophys., 424, 603

Nisini, B., Bacciotti, F., Giannini, T., Massi, F., Eislöffel, J., Podio, L., \& Ray, T. P. 2005, Astron. E Astrophys., 441, 159 
Petrov, R. G., et al. 2007, Astron. \& Astrophys., 464, 1

Podio, L., Bacciotti, F., Nisini, B., Eislöffel, J., Massi, F., Giannini, T., \& Ray, T. P. 2006, Astron. ES Astrophys., 456, 189

Porter, J. M., Oudmaijer, R. D., \& Baines, D. 2004, Astron. 63 Astrophys., 428, 327

Pudritz, R. E., Ouyed, R., Fendt, C., \& Brandenburg, A. 2007, Protostars and Planets V, 277

Raga, A. C., de Colle, F., Kajdič, P., Esquivel, A., \& Cantó, J. 2007, Astron. 83 Astrophys., 465, 879

Ray, T. P. 1987, Astron. E Astrophys., 171, 145

Ray, T. P., Mundt, R., Dyson, J. E., Falle, S. A. E. G., \& Raga, A. C. 1996, Astrophys. J., 468, L103

Ray, T., Dougados, C., Bacciotti, F., Eislöffel, J., \& Chrysostomou, A. 2007, Protostars and Planets V, 231

Scholz, A., Jayawardhana, R., \& Brandeker, A. 2005, Astrophys. J., 629, L41

Shu, F. H., Najita, J. R., Shang, H., \& Li, Z.-Y. 2000, Protostars and Planets IV, 789

Snell, R. L., Loren, R. B., \& Plambeck, R. L. 1980, Astrophys. J., 239, L17

Stehlé, C., Ciardi, B., Lebedev, S. V., \& Lery, T. 2005, SF2A-2005: Semaine de l'Astrophysique Francaise, 355

Sublett, S., Knauer, J. P., Igumenshchev, I. V., Frank, A., \& Meyerhofer, D. D. 2007, Astrophys. Space. Sci., 307, 47

Takami, M., Bailey, J., \& Chrysostomou, A. 2003, Astron. \& Astrophys., 397, 675

Tatulli, E., et al. 2007, Astron. \& Astrophys., 464, 55

Whelan, E. T., Ray, T. P., \& Davis, C. J. 2004, Astron. \& Astrophys., 417, 247

Whelan, E. T., Ray, T. P., Bacciotti, F., Natta, A., Testi, L., \& Randich, S. 2005, Nature, 435, 652

Whelan, E. T., Ray, T. P., Randich, S., Bacciotti, F., Jayawardhana, R., Testi, L., Natta, A., \& Mohanty, S. 2007, Astrophys. J., 659, L45

Zinnecker, H., McCaughrean, M. J., \& Rayner, J. T. 1998, Nature, 394, 862 